\title{
First experience with sodium-glucose co-transporter 2 inhibitors in Polish patients with cardiovascular diseases
}

\author{
Ewa Dziewięcka ${ }^{1}$, Mateusz Winiarczyk ${ }^{2}$, Matylda Gliniak², Monika Kaciczak ${ }^{2}$, \\ Sylwia Wiśniowska-Śmiałek ${ }^{1}$, Aleksandra Karabinowska ${ }^{1}$, Katarzyna Holcman ${ }^{1}$, \\ Magdalena Kostkiewicz ${ }^{1}$, Marta Hlawaty ${ }^{1}$, Agata Leśniak-Sobelga ${ }^{1}$, \\ Piotr Podolec ${ }^{1}$, Paweł Rubiś ${ }^{1}$
}

\begin{abstract}
${ }^{1}$ Department of Cardiac and Vascular Diseases, Jagiellonian University Medical College, John Paul II Hospital, Krakow, Poland

'Jagiellonian University Collegium Medicum, Students' Scientific Group at Department of Cardiac and Vascular Diseases, John Paul II Hospital, Krakow, Poland
\end{abstract}

Type 2 diabetes mellitus (DM2) is a serious public health burden, and is one of the prime causes of morbidity and mortality in patients with cardiovascular diseases (CVD), like coronary artery disease (CAD), heart failure (HF), stroke or peripheral artery disease [1-4]. However, until recently, DM2 management with tight glycemic control, has not been associated with any major improvement in terms of survival in patients with CVD. Thus, the recent introduction of a novel class of agents for the treatment of DM2 - sodium-glucose cotransporter 2 inhibitors (SGLT2i) - has proven to be a breakthrough. These inhibitors combine proximal tubule diuretic and osmotic action leading to a reduction in glucose reabsorption, and mild natriuretic and diuretic effects. Following up on these unique effects, several studies showed that treatment with SGLT2i may not only increase the efficacy of standard antidiabetic therapy, but also reduce CVD and HF mortality [5-8].

Until now, the utilization of SGLT2i in Poland has been relatively limited, which has meant there is a lack of experience in their usage, and underutilization in the treatment of patients. Herein, a study with this novel class of drugs is reported in a cohort of 52 Polish DM2 patients with CVD, including a large proportion of patients with $\mathrm{HF}$, all of whom were prescribed SGLT2i.

A retrospective analysis was performed that included 52 diabetic out- or inpatients, who agreed to treatment with SGLT2i between 2017 and 2019. At baseline, all patients underwent a detailed diagnostic work-up: clinical evaluation, blood tests (including NT-proBNP, fasting glucose, HbA1c, cholesterol LDL and creatinine levels), electrocardiogram and echocardiography. Telephone contact or outpatient visits were carried out in December 2019 and January 2020. The investigation conforms to the principles outlined in the Declaration of Helsinki.

All parameters are presented as means \pm standard deviation or counts (percentages) when appropriate. All variables were tested for normal distribution of data with the Shapiro-Wilk test. Comparisons of continuous parameters between patients with and without HF were conducted with t-tests when normality was confirmed, or otherwise, with the Mann-Whitney test; the $\chi^{2}$ test was performed for the comparison of qualitative parameters. All results were considered statistically significant when their p-value was $<0.05$. The Statistica package, version 13.0 (StatSoft, TIBCO Software Inc.), was used for the statistical analysis.

Out of 52 patients $50(96 \%)$ received empagliflozin and 2 (4\%) dapagliflozin. Most patients $(40 ; 77 \%)$ were also treated with metformin, 15 $(29 \%)$ received insulin and $10(19 \%)$ sulphonylurea. Patients were stratified into those with $\mathrm{HF}$ $(36.7 \%)$ and those without HF (16.3\%) (Table 1). Both groups were burdened with a high number

Address for correspondence: Ewa Dziewięcka, MD, Department of Cardiac and Vascular Diseases, John Paul II Hospital, ul. Prądnicka 80, 31-202 Kraków, Poland, tel: +48 1261422 87, e-mail: ewa@dziewiecka.pl 
Table 1. Detailed characteristics of the study population.

\begin{tabular}{|c|c|c|c|}
\hline Parameter & Patients with HF ( $n=36$ ) & Patients without HF $(n=16)$ & $\mathbf{P}$ \\
\hline Age [years] & $64.6 \pm 9.0$ & $60.4 \pm 13.5$ & 0.47 \\
\hline Male & $29(81 \%)$ & $10(63 \%)$ & 0.32 \\
\hline Heart rate $[\mathrm{bpm}]$ & $76.3 \pm 11.0$ & $73.2 \pm 9.9$ & 0.51 \\
\hline Atrial hypertension & $31(86 \%)$ & $14(88 \%)$ & 0.89 \\
\hline Coronary artery disease & $27(75 \%)$ & $10(91 \%)$ & 0.53 \\
\hline Atrial fibrillation & $8(22 \%)$ & $6(38 \%)$ & 0.15 \\
\hline Dyslipidemia & $31(86 \%)$ & $16(100 \%)$ & 0.006 \\
\hline Obesity & $18(58 \%)$ & $9(56 \%)$ & 0.52 \\
\hline Ejection fraction [\%] & $38 \pm 16$ & $57 \pm 8$ & $<0.001$ \\
\hline LVEDD $[\mathrm{mm}]$ & $57 \pm 10$ & $51 \pm 5$ & 0.03 \\
\hline Fasted glucose [mg/dL] & $10.6 \pm 4.5$ & $6.7 \pm 1.7$ & $<0.001$ \\
\hline $\mathrm{HbA} 1 \mathrm{c}[\%]$ & $8.8 \pm 3.1$ & $6.9 \pm 1.6$ & 0.48 \\
\hline Creatinine $[\mathrm{mg} / \mathrm{dL}]$ & $110 \pm 71$ & $83 \pm 11$ & 0.03 \\
\hline LDL cholesterol [mmol/L] & $2.8 \pm 1.1$ & $2.7 \pm 1.4$ & 0.64 \\
\hline Metformin & $27(75 \%)$ & $13(81 \%)$ & 0.86 \\
\hline Insulin & $12(33 \%)$ & $3(19 \%)$ & 0.46 \\
\hline Sulphonylurea & $8(22 \%)$ & $2(12.5 \%)$ & 0.06 \\
\hline
\end{tabular}

All data are presented as means \pm standard deviation or counts (percentages). HF — heart failure; LDL — low density lipoprotein; LV — left ventricular end-diastolic diameter

of CVD and CVD risk factors. Fasting glucose was significantly higher in the HF group; however, HbA1c was similar. Understandably, HF patients had larger left ventricles with much worse systolic function. Following SGLT2i initiation, tolerance was very good and there were no complaints of any immediate side effects.

After a follow-up of $16.3 \pm 23.6$ months, 47 $(90 \%)$ patients were continuing treatment with SGLT2i. The main reason for SGLT2i discontinuation was the price of the medication (no subsidy in the form of a reimbursement was available). Three $(5.8 \%)$ patients reported benign urinary tract infection (uncomplicated cystitis or urethritis) during the course of treatment, which resolved itself without major incident (no prolonged antibiotic therapy or hospitalization were required). As for the HF group, their New York Heart Association (NYHA) class improved (baseline: $2.3 \pm 0.65 \mathrm{vs.}$ follow-up: $2.0 \pm 0.67 ; \mathrm{p}=0.04$ ), patients reported sustained weight-loss of $4.5 \pm 5.6 \mathrm{~kg}$, and required lower daily furosemide dosage (baseline: $56.7 \pm$ \pm 20.7 vs. follow-up: $46.7 \pm 53.6 \mathrm{mg} /$ day; $\mathrm{p}=0.03$ ).

Type 2 diabetes mellitus and CVD, especially $\mathrm{CAD}$ and $\mathrm{HF}$, independently contribute to cardiovascular morbidity $[2,3,9]$. They frequently coexist - DM2 is present in up to $45 \%$ of $\mathrm{HF}$ patients - and HF death is one of the most common causes of death among patients with both CVD and DM2 [3, 10]. Numerous studies published in the last decade have reported on negligible improvement in terms of mortality rates in patients with DM2 and coexisting CVDs. It is only recently that large outcome trials have demonstrated the unprecedented efficacy of SGLT2i in the reduction of major cardiovascular events $[5,6]$. Furthermore, it seems that we are on the verge of a paradigm shift in the management of DM2 (with and without CVDs). Therefore, the SGLT2i are a class I recommendation for DM2 patients with high CVD risk in the latest guidelines from the European Society of Cardiology 2019, especially with poor DM2 control [3].

Presented herein, are the first Polish results of treatment with SGLT2i. Overall, there was a very good uptake of this new therapy; in the present study, the SGLT2i was mostly introduced in high-risk patients with CVD which had already been established, of whom a large proportion consisted of HF patients. As for side effects, a very small number of urinary tract infections (an anticipated and previously reported problem due to glucosuria) occurred during the course of treatment (a treatment lasting for more than 2 years for some patients). However, it was slightly more prevalent than in other studies presented $(1.5 \%$ in 
dapagliflozin and $1.7 \%$ in empagliflozin analysis) $[5,6]$. Moreover, in contrast to those studies there were no genital infections observed in the current population. Reassuringly, substantial improvements were observed in HF patients in terms of symptoms and physical performance (lower NYHA class), sustainable weight reduction, along with a simultaneous reduction in furosemide daily dosage.

Initial experience with a new class of antidiabetic drugs - SGLT2i is very positive and shows results similar to those reported in major trials and registries. The sustainable benefit in the HF subgroup is of particular importance as these patients are particularly prone to complications. As it has recently been announced that the cost of SGLT2i can be reimbursed in Poland (with the application of strict criteria for eligibility), there now seems to be a ray of hope on the horizon for patients with DM2 and CVDs.

\section{Acknowledgements}

This work was supported by the Medical College, Jagiellonian University (grant number SAP N41/DBS/000130).

\section{Conflict of interest: None declared}

\section{References}

1. Lenzen M, Ryden L, Ohrvik J, et al. Euro Heart Survey Investigators. Diabetes known or newly detected, but not impaired glucose regulation, has a negative influence on 1-year outcome in patients with coronary artery disease: a report from the Euro Heart Survey on diabetes and the heart. Eur Heart J. 2006;
27(24): 2969-2974, doi: 10.1093/eurheartj/ehl363, indexed in Pubmed: 17090612.

2. Gasior M, Pres D, Stasik-Pres G, et al. Effect of blood glucose levels on prognosis in acute myocardial infarction in patients with and without diabetes, undergoing percutaneous coronary intervention. Cardiol J. 2008; 15(5): 422-430, indexed in Pubmed: 18810716 .

3. Cosentino F, Grant PJ, Aboyans V, et al. ESC Scientific Document Group. 2019 ESC Guidelines on diabetes, pre-diabetes, and cardiovascular diseases developed in collaboration with the EASD. Eur Heart J. 2020; 41(2): 255-323, doi: 10.1093/eurheartj/ ehz486, indexed in Pubmed: 31497854.

4. Kralev S, Krause B, Papavassiliu T, et al. Clinical outcome of patients with diabetes presenting with ST-elevation myocardial infarction and treated with concomitant use of glycoprotein IIb/ IIIa inhibitors. Cardiol J. 2009; 16(3): 234-240, indexed in Pubmed: 19437397.

5. Zinman B, Wanner C, Lachin JM, et al. EMPA-REG OUTCOME Investigators. Empagliflozin, Cardiovascular Outcomes, and Mortality in Type 2 Diabetes. N Engl J Med. 2015; 373(22): 2117-2128, doi: 10.1056/NEJMoa1504720, indexed in Pubmed: 26378978.

6. Wiviott SD, Raz I, Bonaca MP, et al. Dapagliflozin and cardiovascular outcomes in type 2 diabetes. N Engl J Med. 2019; 380(19): 1880-1882, doi: 10.1056/nejmc1902837.

7. Fitchett D, Zinman B, Wanner C, et al. Heart failure outcomes with empagliflozin in patients with type 2 diabetes at high cardiovascular risk: results of the EMPA-REG OUTCOME® trial. Eur Heart J. 2016; 37(19): 1526-1534, doi: 10.1093/eurheartj/ehv728, indexed in Pubmed: 26819227.

8. McMurray JJV, Solomon SD, Inzucchi SE, et al. Dapagliflozin in patients with heart failure and reduced ejection fraction. $\mathrm{N}$ Engl J Med. 2020; 382(10): 972-973, doi: 10.1056/nejmc1917241.

9. Di Angelantonio E, Kaptoge S, Wormser D, et al. Association of cardiometabolic multimorbidity with mortality. JAMA. 2015; 314(1): 52-60, doi: 10.1001/jama.2015.7008, indexed in Pubmed: 26151266.

10. Sharma A, Zhao X, Hammill B, et al. Trends in noncardiovascular comorbidities among patients hospitalized for heart failure. Circ Hear Fail. 2018; 11(6), doi: 10.1161/circheartfailure.117.004646. 